\title{
Temper Tantrums in Young Children
}

\author{
Dana H. Davidson \\ Department of Family and Consumer Sciences
}

A temper tantrum is a violent outburst of anger. Anger is a basic human emotion that is manifested early in infancy and continues throughout the life span. Anger is a normal reaction to frustration, fear, or other stress. Some children seem more angry than others early on, but their anger should diminish as they learn to cope with the world. During early childhood, children often have fits of anger that seem volcanic in intensity. Their rage may include behaviors such as screaming, cursing, breaking things, rolling on the floor, crying loudly, hitting, or running around the room. They may even vomit, hold their breath, hit their head, or run off to hide.

Tantrums seem to appear in stages, with an early warning of "clouds on the horizon." This is followed by the actual tantrum, which usually lasts minutes and may seem an eternity to the adult in charge. Finally, after the tantrum is a period of "hangover," when the child is tired, red-faced, runny-nosed, and needing to be held. Often after this last stage children are ready to continue on as if nothing has happened!

There are ways to prevent tantrums, and there are ways to deal with them when they occur. One of the most important things for the adult to know is not to get caught up in the child's anger-this will make the problem last longer into childhood. Providing the model of proper human emotions is very important to the child. If you spot the storm signals, such as increased noise level, red face, and brow-lowering, try to change the situation before the tantrum occurs. During the tantrum, it is suggested that adults remain nearby and provide neutral or warm support to the child. That is, ignore the tantrum behaviors, but let the child know by your presence that you are available for help.
During the tantrum is not a time for discipline, for this is an emotional outburst that does not leave much energy for learning. After the tantrum, however, adults can provide words to the child to help learn why there was anger and how it felt. If the tantrum occurs in public, and the parent is embarrassed, it works to sit or remain near the child, smile to passers-by, and let others know you are in control even though the child is not. Lack of reinforcement usually shortens the tantrum. After the tantrum, continue on with the public activity. If the tantrum occurs at home, it is possible for the adult to move away out of sight of the child yet remain nearby. Some psychologists recommend isolation or "time out" as a consequence of a tantrum.

Provide rewards for non-angry behavior. Try to figure out the causes of the child's anger. Sometimes illness, hunger, and fatigue may be causes. Frustration is a common reason for tantrums, so try to reduce the causes of frustration. Providing language is an important tool for children to use for managing their emotions. Know that tantrums peak at age four and may occur to age six before they diminish. Some tantrums may be expected of all children. Our goal as parents and caretakers is to help children learn to recognize and deal with their emotions so that tantrums become a behavior only found in young children-never in adults.

\section{References}

Schaefer, C., and H.L. Millman. 1981. How to help children with common problems. Van Nostrand Reinhold Co.

Keiki 'O Hawai' 'i. Cooperative Extension Service, College of Tropical Agriculture and Human Resources, University of Hawaii, in cooperation with the Hawaii Department of Health, Children's Mental Health Services Branch. 